\title{
SOME TRACE CLASS COMMUTATORS OF TRACE ZERO
}

\author{
FUAD KITTANEH
}

(Communicated by Palle E. T. Jorgensen)

\begin{abstract}
It is shown that if $T$ is an operator on a separable complex Hilbert space and $X$ is a Hilbert-Schmidt operator such that $T X-X T$ is a trace class operator, then the trace of $T X-X T$ is zero provided one of the two conditions holds: (a) $T^{2}$ is normal; (b) $T^{n}$ is normal for some integer $n>2$ and $T^{*} T-T T^{*}$ is a trace class operator. Related results involving essentially unitary operators and Cesàro operators are also given.
\end{abstract}

\section{INTRODUCTION}

In their work on the traces of commutators of integral operators, Helton and Howe [8, Lemma 1.3] proved that if $A$ is a selfadjoint operator on a separable complex Hilbert space and $X$ is a compact operator such that the commutator $A X-X A$ is in the trace class, then the trace of $A X-X A$ is equal to zero. In [18, Lemma 8] Weiss proved that if $N$ is a normal operator and $X$ is a Hilbert-Schmidt operator such that $N X-X N$ is in the trace class, then the trace of $N X-X N$ is also zero. These results have been extended to certain nonnormal operators in [10]; also quantitative versions of these results have been obtained in [9] and [11].

The purpose of this paper is to pursue our investigation of the trace vanishing phenomenon of certain trace class commutators. In $\S 2$ we improve the previously known results [10, Theorems 3 and 4] about zero-trace commutators involving square roots of normal operators. Zero-trace commutators involving essentially unitary operators and Cesàro operators are discussed in $\S 3$.

Let $B(H)$ denote the algebra of all bounded linear operators acting on a separable complex Hilbert space $H$. Let $C_{1}(H)$ denote the ideal of trace class operators in $B(H)$. If $T \in C_{1}(H)$ or $T \in B(H)$ is positive and if $\left\{f_{n}\right\}$ is an orthonormal basis for $H$, then the trace of $T$, denoted by $\operatorname{tr} T$ and defined by $\operatorname{tr} T=\sum_{n}\left(T f_{n}, f_{n}\right)$, is independent of the choice of $\left\{f_{n}\right\}$. A compact

Received by the editors March 20, 1990 and, in revised form, December 19, 1990.

1980 Mathematics Subject Classification (1985 Revision). Primary 47B05, 47B10, 47B15, 47B37, 47B47; Secondary 47B20.

Key words and phrases. Commutator, compact operator, Hilbert-Schmidt operator, trace, trace class, Schatten $p$-class, normal operator, selfadjoint operator, essentially unitary operator, Cesàro operator. 
operator $T \in B(H)$ is said to be in the Schatten $p$-class $C_{p}(H) \quad(1 \leq p \leq$ $\infty)$ if $\operatorname{tr}|T|^{p}<\infty$, where $|T|=\left(T^{*} T\right)^{1 / 2}$ is the absolute value of $T$. It is known that $C_{p}(H) \quad(1 \leq p \leq \infty)$ becomes a Banach space under the norm $\|T\|_{p}=\left(\operatorname{tr}|T|^{p}\right)^{1 / p}$. Note that $C_{2}(H)$ is the ideal of Hilbert-Schmidt operators and $C_{\infty}(H)$ is the ideal of compact operators with $\|\cdot\|_{\infty}$ denoting the usual operator norm. If $X$ and $Y$ are in $B(H)$ such that both $X Y$ and $Y X$ lie in $C_{1}(H)$, then $\operatorname{tr}(X Y)=\operatorname{tr}(Y X)$, (see [16, Corollary 3.8] or [14, Lemma 2.1]). In particular if $X \in C_{p}(H)$ and $Y \in C_{p}(H)$, where $1 / p+1 / q=1$, then $X Y$ and $Y X$ lie in $C_{1}(H)$ and $\operatorname{tr}(X Y)=\operatorname{tr}(Y X)$. If $X$ and $Y$ are in $B(H)$ such that $0 \leq X \leq Y$ and if $Y \in C_{p}(H)$, then $X \in C_{p}(H)$. These definitions and properties can be carried over to the spaces $B\left(H_{1}, H_{2}\right)$ and $C_{p}\left(H_{1}, H_{2}\right)$ of operators from a Hilbert space $H_{1}$ into a Hilbert space $H_{2}$. We refer the reader to $[6,16]$ for further properties of the Schatten $p$-classes.

\section{ROOTS OF NORMAL OPERATORS}

Utilizing a result of Radjavi and Rosenthal [15, Theorem 1] concerning the structure of the square roots of normal operators, the author proved in [10, Theorem 3] that if $T \in B(H)$ with $T^{2}$ normal, $T^{*} T-T T^{*} \in C_{1}(H)$, and if $X \in C_{2}(H)$ such that $T X-X T \in C_{1}(H)$, then $\operatorname{tr}(T X-X T)=0$. This generalizes Weiss' result [18, Theorem 8]. Using a structure result due to Gilfeather enables us to prove a general result of this sort that is valid for arbitrary $n$th roots of normal operators. Moreover if $n=2$, then we show that the assumption $T^{*} T-T T^{*} \in C_{1}(H)$ can be removed. To achieve our goal we need some preliminary results.

Lemma 1. If $T \in B(H)$ is nilpotent, i.e., $T^{n}=0$ for some integer $n>1$, and if $T^{*} T-T T^{*} \in C_{p}(H)$ for some $p, 1 \leq p \leq \infty$, then $T \in C_{2 p}(H)$.

Proof. Since $T^{n}=0$, it follows by Theorem 1 in [7] that there exists a decomposition $H=\bigoplus_{i=1}^{n} H_{i}$ of $H$ into the direct sum of an orthogonal family of subspaces $H_{1}, H_{2}, \ldots, H_{n}$ such that the matrix of $T$ corresponding to this decomposition has the upper triangular form

$$
T=\left[\begin{array}{cccc}
0 & T_{12} & \cdots & T_{1 n} \\
0 & 0 & & \vdots \\
\vdots & & \ddots & T_{n-1, n} \\
0 & 0 & \cdots & 0
\end{array}\right] .
$$

Since $T T^{*}-T^{*} T \in C_{p}(H)$, it follows that every $(i, j)$ entry of the operator matrix $T T^{*}-T^{*} T$ is in $C_{p}\left(H_{j}, H_{i}\right) \quad(1 \leq i, j \leq n)$. The $(1,1)$ entry of this matrix is $\left|T_{12}^{*}\right|^{2}+\left|T_{13}^{*}\right|^{2}+\cdots+\left|T_{1 n}^{*}\right|^{2} \in C_{p}\left(H_{1}\right)$. Since $\left|T_{12}^{*}\right|^{2}+\left|T_{13}^{*}\right|^{2}+\cdots+\left|T_{1 n}^{*}\right|^{2} \geq$ $\left|T_{1 j}^{*}\right|^{2} \geq 0$ for $2 \leq j \leq n$, it follows that $\left|T_{1 j}^{*}\right|^{2} \in C_{p}\left(H_{1}\right)$ for $2 \leq j \leq n$. Hence $T_{1 j}^{*} \in C_{2 p}\left(H_{1}, H_{j}\right)$, and so $T_{1 j} \in C_{2 p}\left(H_{j}, H_{1}\right)$ for $2 \leq j \leq n$. The $(2,2)$ entry of $T T^{*}-T^{*} T$ is $\left|T_{23}^{*}\right|^{2}+\cdots+\left|T_{2 n}^{*}\right|^{2}-\left|T_{12}\right|^{2} \in C_{p}\left(H_{2}\right)$. Since $\left|T_{12}\right|^{2} \in C_{p}\left(H_{2}\right)$, 
it follows that $\left|T_{23}^{*}\right|^{2}+\cdots+\left|T_{2 n}^{*}\right|^{2} \in C_{p}\left(H_{2}\right)$. Hence $T_{2 j} \in C_{2 p}\left(H_{j}, H_{2}\right)$ for $3 \leq j \leq n$. By repeating this argument we can show that $T_{i j} \in C_{2 p}\left(H_{j}, H_{i}\right)$ for $1 \leq i, j \leq n$. Therefore $T \in C_{2 p}(H)$, as required.

Lemma 2. Let $T \in B(H)$ be similar to a normal operator $N \in B(H)$. If $X \in C_{2}(H)$ and $T X-X T \in C_{1}(H)$, then $\operatorname{tr}(T X-X T)=0$.

Proof. Assume that $T=P^{-1} N P$ for some invertible operator $P \in B(H)$. Now

$$
\begin{aligned}
T X-X T & =P^{-1} N P X-X P^{-1} N P \\
& =P^{-1}\left(N P X P^{-1}-P X P^{-1} N\right) P \\
& =P^{-1}(N Y-Y N) P, \quad \text { where } Y=P X P^{-1} \in C_{2}(H) .
\end{aligned}
$$

Therefore $N Y-Y N \in C_{1}(H)$, and so by Weiss' result $\operatorname{tr}(N Y-Y N)=0$. Because similarity preserves the trace (see [6, Corollary 8.1]), it follows that $\operatorname{tr}(T X-X T)=\operatorname{tr}\left(P^{-1}(N Y-Y N) P\right)=\operatorname{tr}(N Y-Y N)=0$.

Lemma 3. Let $T \in B(H)$ with $T^{2}=0$. If $X \in B(H)$ and $T X-X T \in C_{1}(H)$, then $\operatorname{tr}(T X-X T)=0$.

Proof. Since $T^{2}=0$, it follows that $H$ can be decomposed as $H=H_{1} \oplus H_{2}$ for some orthogonal subspaces $H_{1}, H_{2}$ and with respect to this decomposition $T=\left[\begin{array}{cc}0 & T_{12} \\ 0 & 0\end{array}\right]$. Now if $X=\left[\begin{array}{ll}X_{11} & X_{12} \\ X_{21} & X_{22}\end{array}\right]$ is the operator matrix of $X$ corresponding to the above decomposition of $H$, then

$$
T X-X T=\left[\begin{array}{cc}
T_{12} X_{21} & T_{12} X_{22}-X_{11} T_{12} \\
0 & -X_{21} T_{12}
\end{array}\right] .
$$

Since $T X-X T \in C_{1}(H)$, it follows that $T_{12} X_{21} \in C_{1}\left(H_{1}\right)$ and $X_{21} T_{12} \in$ $C_{1}\left(H_{1}\right)$, and so by Corollary 3.8 in [16], we have

$$
\operatorname{tr}(T X-X T)=\operatorname{tr}\left(T_{12} X_{21}\right)-\operatorname{tr}\left(X_{21} T_{12}\right)=0 .
$$

Now we are in a position to establish the main results of this section.

Theorem 4. Let $T \in B(H)$ be such that $T^{n}$ is normal for some integer $n \geq 2$ and $T^{*} T-T T^{*} \in C_{1}(H)$. If $X \in C_{2}(H)$ and $T X-X T \in C_{1}(H)$, then $\operatorname{tr}(T X-X T)=0$.

Proof. Since $T^{n}$ is normal, it follows from a result of Gilfeather [5, Theorem 3.1] that there exist reducing subspaces $\left\{H_{i}\right\}_{i=0}^{\infty}$ for $T$ such that $H=\bigoplus_{i=0}^{\infty} H_{i}$, $T_{0}=T \mid H_{0}$ is nilpotent with $T_{0}^{n}=0$, and $T_{i}=T \mid H_{i}$ is similar to a normal operator $N_{i}$ for $i=1,2, \ldots$. Let $X=\left[X_{i j}\right]$ be the matrix representation of $X$ with respect to the above decomposition of $H$. Since $T=\bigoplus_{i=0}^{\infty} T_{i}$, it follows that the $(i, i)$ entry of $T X-X T$ is $T_{i} X_{i i}-X_{i i} T_{i}$ for $i=0,1,2, \ldots$. Since $T X-X T \in C_{1}(H)$ and $X \in C_{2}(H)$, it follows that $T_{i} X_{i i}-X_{i i} T_{i} \in C_{1}\left(H_{i}\right)$ and $X_{i i} \in C_{2}\left(H_{i}\right)$ for $i=0,1,2 \ldots$ (see [6, p. 94]). Similarly $T^{*} T-T T^{*} \in$ $C_{1}(H)$ implies that $T_{0}^{*} T_{0}-T_{0} T_{0}^{*} \in C_{1}\left(H_{0}\right)$. Hence by Lemma $1, T_{0} \in C_{2}\left(H_{0}\right)$, and so $\operatorname{tr}\left(T_{0} X_{00}\right)=\operatorname{tr}\left(X_{00} T_{0}\right)$. By Lemma 2, we also have $\operatorname{tr}\left(T_{i} X_{i i}-X_{i i} T_{i}\right)=0$ 
for $i=1,2, \ldots$. Therefore $\operatorname{tr}(T X-X T)=\sum_{i=0}^{\infty} \operatorname{tr}\left(T_{i} X_{i i}-X_{i i} T_{i}\right)=0$, as desired.

Following the proof of Theorem 4 and using Lemma 3 instead of Lemma 1, we now improve Theorems 3 and 4 in [10] as follows.

Theorem 5. Let $T \in B(H)$ with $T^{2}$ normal. If $X \in C_{2}(H)$ and $T X-X T \in$ $C_{1}(H)$, then $\operatorname{tr}(T X-X T)=0$.

Proof. With the same notations as in the proof of Theorem 4, we have that $\operatorname{tr}\left(T_{i} X_{i i}-X_{i i} T_{i}\right)=0$ for $i=1,2, \ldots$. Since $T_{0}^{2}=0$ and $T_{0} X_{00}-X_{00} T_{0} \in$ $C_{1}\left(H_{0}\right)$, it follows by Lemma 3 that $\operatorname{tr}\left(T_{0} X_{00}-X_{00} T_{0}\right)=0$. So $\operatorname{tr}(T X-X T)=$ $\sum_{i=0}^{\infty} \operatorname{tr}\left(T_{i} X_{i i}-X_{i i} T_{i}\right)=0$ and the proof is complete.

We conclude this section with the following remarks.

1. If $T^{2}=0$, then it follows from Lemma 1 and the basic properties of the Schatten $p$-norms that $\left\|T^{*} T-T T^{*}\right\|_{p}=2^{1 / p}\|T\|_{2 p}^{2}$ for $1 \leq p \leq \infty$. Moreover, if $T^{n}=0$ for some integer $n>2$, then estimates relating $\left\|T^{*} T-T T^{*}\right\|_{p}$ and $\|T\|_{2 p}$ can be obtained using some recent inequalities for the Schatten $p$-norms of partitioned operator matrices [3].

It should be noted that for $p=\infty$, Lemma 1 remains true under the weaker assumption that $T$ is quasinilpotent. To see this, one needs to formulate the problem in the Calkin algebra setting. However, it is not known to the author whether Lemma 1 remains true for $p \neq \infty$ under the quasinilpotency assumption. A well-known, related result in this direction asserts that if $T$ is quasinilpotent such that $T-T^{*} \in C_{p}(H)$ for some $p, 1<p \leq \infty$, then $T \in C_{p}(H)$ (see [6, p. 215]).

2. It follows from the structure theorem of Radjavi and Rosenthal [15, Theorem 1] that if $T^{2}$ is normal and if $T^{*} T-T T^{*} \in C_{p}(H)$ for some $p$, $1 \leq p \leq \infty$, then $T=N+K$, where $N$ is normal and $K \in C_{2 p}(H)$ with $N K=-K N$ (see the proof of Theorem 3 in [10]). Also it follows from Lemma 1 and from a result of Apostol [1] that if $T^{n}$ and $T^{m}$ are normal for some relatively prime integers $n, m$ with $m>n \geq 2$ and if $T^{*} T-T T^{*} \in C_{p}(H)$ for some $p, 1 \leq p \leq \infty$, then $T=N+K$, where $N$ is normal, $K \in C_{2 p}(H)$, $K^{n}=0$, and $N K=K N=0$.

3. If $T^{n}$ is normal for some integer $n>2$ and $T$ is invertible, then by a result of Stampfli [17], $T$ is similar to a normal operator. Thus if $X \in C_{2}(H)$ and $T X-X T \in C_{1}(H)$, then $\operatorname{tr}(T X-X T)=0$ by Lemma 2 .

\section{EsSentially unitary operators AND Cesàro operators}

Recall that an operator $A \in B(H)$ is said to be essentially unitary if $\pi(A)$ is unitary, where $\pi$ is the canonical map of $B(H)$ onto the Calkin algebra $B(H) / C_{\infty}(H)$. Equivalently, $A$ is essentially unitary if both $1-A^{*} A$ and $1-A A^{*}$ are in $C_{\infty}(H)$.

If $A \in B(H)$ and $X \in C_{\infty}(H)$ such that both $A X-X A$ and $A^{*} X-X A^{*}$ are in $C_{1}(H)$, then it follows by the Helton-Howe lemma [8, Lemma 1.3] that 
$\operatorname{tr}(A X-X A)=0$ (see also [19]). Consequently if $A \in B(H)$ is an isometry of finite multiplicity (in particular if $A$ is unitary) and if $X \in C_{\infty}(H)$ and $A X-X A \in C_{1}(H)$, then $\operatorname{tr}(A X-X A)=0$ (see [10, Theorems 2 and 5]).

In the same spirit, we have the following more general result.

Theorem 6. Let $A \in B(H)$ be such that both $1-A^{*} A$ and $1-A A^{*}$ are in $C_{p}(H)$ for some $p, 1 \leq p \leq \infty$. If $X \in C_{q}(H)$, where $1 / p+1 / q=1$, and if $A X-X A \in C_{1}(H)$, then $\operatorname{tr}(A X-X A)=0$.

Proof. By the Helton-Howe lemma, it is sufficient to show that $A^{*} X-X A^{*} \in$ $C_{1}(H)$. Assume that $A^{*} A=1+K_{1}$ and $A A^{*}=1+K_{2}$, where $K_{1}$ and $K_{2}$ are both in $C_{p}(H)$. Since $A X-X A \in C_{1}(H)$, it follows that $A^{*}(A X-X A) A^{*} \in$ $C_{1}(H)$, and so $\left(1+K_{1}\right) X A^{*}-A^{*} X\left(1+K_{2}\right) \in C_{1}(H)$. In view of the fact that $K_{1} X$ and $X K_{2}$ are both in $C_{1}(H)$, it now follows that $A^{*} X-X A^{*} \in C_{1}(H)$, which is the desired conclusion.

For an account on essentially unitary operators, the reader is referred to [13] and references therein.

As an application of Theorem 6 we now discuss commutators involving Cesàro operators.

The discrete Cesàro operator $K_{0}$ acting on the Hilbert space $l^{2}$ of square summable complex sequences is defined by

$$
K_{0}\left(a_{1}, a_{2}, a_{3}, \ldots\right)=\left(a_{1}, \frac{a_{1}+a_{2}}{2}, \frac{a_{1}+a_{2}+a_{3}}{3}, \ldots\right) .
$$

With respect to the standard orthonormal basis $\left\{e_{n}\right\}$ for $l^{2}$, the matrix of $K_{0}$ is given by

$$
\left[\begin{array}{cccc}
1 & 0 & 0 & \cdots \\
\frac{1}{2} & \frac{1}{2} & 0 & \cdots \\
\frac{1}{3} & \frac{1}{3} & \frac{1}{3} & \cdots \\
\vdots & \vdots & \vdots & \ddots
\end{array}\right] .
$$

Two continuous analogs of $K_{0}$ are the Cesàro operators $K_{1}$ and $K_{\infty}$ defined on the Hilbert spaces $L^{2}(0,1)$ and $L^{2}(0, \infty)$, respectively, by

$$
\left(K_{1} f\right)(x)=\frac{1}{x} \int_{0}^{x} f(y) d y \text { for } 0<x<1
$$

and

$$
\left(K_{\infty} f\right)(x)=\frac{1}{x} \int_{0}^{x} f(y) d y \text { for } 0<x<\infty .
$$

The basic properties of these operators were first discussed by Brown, Halmos, and Shields in [4]. It was shown in [4, Theorems 3, 4, and 5] that $K_{0}$ is a hyponormal operator, $1-K_{1}^{*}$ is the unilateral shift operator on $L^{1}(0,1)$, and $1-K_{\infty}^{*}$ is the bilateral shift operator on $L^{2}(0, \infty)$. In [12, Theorem 4] it was shown that $K_{0}$ is in fact subnormal. 
Using the fact that $1-K_{1}^{*}$ is the unilateral shift operator on $L^{2}(0,1)$, we can easily show that if $X \in C_{\infty}\left(L^{2}(0,1)\right)$ such that $K_{1} X-X K_{1} \in C_{1}\left(L^{2}(0,1)\right)$, then $K_{1}^{*} X-X K_{1}^{*} \in C_{1}\left(L^{2}(0,1)\right)$ and so $\operatorname{tr}\left(K_{1} X-X K_{1}\right)=0$. Note that a similar result for the operator $K_{\infty}$ can be stated in the obvious way.

It is worth while to remark that as in the continuous cases, the operator $1-K_{0}$ is essentially unitary. In fact it has been shown in [4] that if $T=1-K_{0}$, then $1-T T^{*}$ is the diagonal operator defined by

$$
\left(1-T T^{*}\right) e_{n}=\frac{1}{n} e_{n} \text { for } n=1,2, \ldots
$$

Consequently, $1-T T^{*} \in C_{p}\left(l^{2}\right) \quad(1<p \leq \infty)$. Since $T$ is a hyponormal contraction (in fact $\|T\|=1$; see [4]) it follows that $0 \leq 1-T^{*} T \leq 1-T T^{*}$ and so $1-T^{*} T \in C_{p}\left(l^{2}\right) \quad(1<p \leq \infty)$. Thus it follows easily from Theorem 6 that if $X \in C_{q}\left(l^{2}\right)$ for some $q, 1 \leq q<\infty$ and if $K_{0} X-X K_{0} \in C_{1}\left(l^{2}\right)$, then $\operatorname{tr}\left(K_{0} X-X K_{0}^{q}\right)=0$.

Another relevant property of the Cesàro operator $K_{0}$ that deserves attention is that $K_{0}^{*} K_{0}-K_{0} K_{0}^{*} \in C_{1}\left(l^{2}\right)$. This can be seen via the Berger-Shaw result [2, Theorem 1], since $K_{0}$ is a hyponormal operator with a cyclic vector (see [12, Theorem 1]). More simply, $K_{0}^{*} K_{0}-K_{0} K_{0}^{*} \in C_{1}\left(l^{2}\right)$ follows from the fact that $K_{0}^{*} K_{0}-K_{0} K_{0}^{*}$ is a positive operator with a finite trace. Indeed $\operatorname{tr}\left(K_{0}^{*} K_{0}-K_{0} K_{0}^{*}\right)=1$. To see this, observe that $K_{0} e_{n}=\sum_{j=n}^{\infty} \frac{1}{j} e_{j}$ and $K_{0}^{*} e_{n}=$ $\frac{1}{n} \sum_{j=1}^{n} e_{j}$ for $n=1,2, \ldots$. Thus

$$
\begin{aligned}
\left(\left(K_{0}^{*} K_{0}-K_{0} K_{0}^{*}\right) e_{n}, e_{n}\right) & =\left\|K_{0} e_{n}\right\|^{2}-\left\|K_{0}^{*} e_{n}\right\|^{2} \\
& =\left(\sum_{j=n}^{\infty} \frac{1}{j^{2}}\right)-\frac{1}{n} \text { for } n=1,2, \ldots .
\end{aligned}
$$

If $s_{n}=\sum_{m=1}^{n}\left(\left(K_{0}^{*} K_{0}-K_{0} K_{0}^{*}\right) e_{m}, e_{m}\right)$ for $n=1,2, \ldots$, then it is not difficult to see that

$$
s_{n}=\sum_{j=1}^{\infty} \frac{n}{(j+n)^{2}} \text { for } n=1,2, \ldots .
$$

Using the facts that

$$
\sum_{j=1}^{\infty} \frac{1}{(j+n)^{2}}=\int_{0}^{1} \frac{x^{n} \log x}{x-1} d x
$$

and

$$
1<\frac{\log x}{x-1}<\frac{1}{\sqrt{x}} \text { for } 0<x<1,
$$

we obiain that

$$
n \int_{0}^{1} x^{n} d x<s_{n}<n \int_{0}^{1} x^{n-1 / 2} d x
$$

hence $\frac{n}{n+1}<s_{n}<\frac{n}{n+1 / 2}$ for $n=1,2, \ldots$. Consequently, $\operatorname{tr}\left(K_{0}^{*} K_{0}-K_{0} K_{0}^{*}\right)$ $=\lim _{n \rightarrow \infty} s_{n}=1$. 
Finally, we would like to ask the following question concerning the Cesàro operator $K_{0}$.

Question. Is it true that $\operatorname{tr}\left(K_{0} X-X K_{0}\right)=0$ whenever $X \in C_{\infty}\left(l^{2}\right)$ and $K_{0} X-$ $X K_{0} \in C_{1}\left(l^{2}\right)$ ?

\section{ACKNOWLEDGMENT}

The author is grateful to the referee for many useful remarks.

\section{REFERENCES}

1. C. Apostol, Propriétés de certain operateurs bornes des escapes de Hilbert, Rev. Roumaine Math. Pures Appl. 10 (1965), 643-644.

2. C. A. Berger and B. I. Shaw, Self-commutators of multicyclic hyponormal operators are always trace class, Bull. Amer. Math. Soc. 79 (1973), 1193-1199.

3. R. Bhatia and F. Kittaneh, Norm inequalities for partitioned operators and an application, Math. Ann. 287 (1990), 719-726.

4. A. Brown, P. R. Halmos, and A. L. Shields, Cesàro operators, Acta Sci. Math. (Szeged) 26 (1965), 125-137.

5. F. Gilfeather, Operator valued roots of abelian analytic functions, Pacific J. Math. 55 (1974), 127-148.

6. I. C. Gohberg and M. G. Krein, Introduction to the theory of linear nonselfadjoint operators, Transl. Math. Mono., Amer. Math. Soc., Providence, RI, 1969.

7. P. R. Halmos, Capacity in Banach algebras, Indiana Univ. Math. J. 20 (1971), 855-863.

8. J. Helton and R. Howe, Traces of commutators of integral operators, Acta Math. 135 (1975), 271-305.

9. F. Kittaneh, Inequalities for the Schatten p-norm, Glasgow Math. J. 26 (1985), 141-143.

10. __, On zero-trace commutators, Bull. Austral. Math. Soc. 34 (1986), 119-126.

11. __ Inequalities for the Schatten p-norm II, Glasgow Math. J. 29 (1987), 99-104.

12. T. L. Kriete, III and D. Trutt, The Cesàro operator in $l^{2}$ is subnormal, Amer. J. Math. 93 (1971), 215-225.

13. R. Lang, Sets of essentially unitary operators, Trans. Amer. Math. Soc. 281 (1984), 65-74.

14. C. Laurie, E. Nordgren, H. Radjavi, and P. Rosenthal, On triangularization of algebras of operators, J. Reine Angew. Math. 327 (1981), 143-155.

15. H. Radjavi and P. Rosenthal, On roots of normal operators, J. Math. Anal. Appl. 34 (1971), 653-664.

16. B. Simon, Trace ideals and their applications, Cambridge Univ. Press, 1979.

17. J. G. Stampfli, Roots of scalar operators, Proc. Amer. Math. Soc. 13 (1962), 796-798.

18. G. Weiss, The Fuglede commutativity theorem modulo the Hilbert-Schmidt class and generating functions for matrix operators. I, Trans. Amer. Math. Soc. 246 (1978), 193-209.

19. __ The Fuglede commutativity theorem modulo operator ideals, Proc. Amer. Math. Soc. 83 (1981), 113-118.

Department of Mathematics, Indiana University, Bloomington, Indiana 47405

Current address: Department of Mathematics, University of Jordan, Amman, Jordan 